\author{
Stefan Kühl
}

\title{
Die Internationalität der Rassenforschung im 20. Jahrhundert
}

\begin{abstract}
Stefan Kühl ist Soziologe. Er ist Professor für Soziologie mit dem Schwerpunkt Organisationssoziologie an der Universität Bielefeld. In einem seiner aktuellen Forschungsprojekte beschäftigt er sich mit dem Genozid aus organisationssoziologischer Perspektive. Zu seinen thematisch relevanten Publikationen gehören u.a. Ganz normale Organisationen. Zur Soziologie des Holocaust (2014) und Die Internationale der Rassisten. Der Aufstieg und Niedergang der internationalen Bewegung für Eugenik und Rassenhygiene im zwanzigsten Jahrhundert (1997). ${ }^{1}$
\end{abstract}

Die immer noch dominierende Geschichtsschreibung über Eugenik und Rassenhygiene in Europa und den USA zeichnet ein karges Bild des Zusammenhangs von Wissenschaftlichkeit und Rassismus: Gerade US-amerikanische Historiker präsentieren eine Gruppe von rassistischen, wissenschaftlich umstrittenen Eugenikern als den Hauptstrom der eugenischen Bewegung, der von der Gründung der ersten eugenischen Organisationen am Anfang des 20. Jahrhunderts bis in die 1930er-Jahre die Diskussion beherrscht habe. Diese Gruppe, so die Meinung jener Historiker, sei dann besonders in den USA und Großbritannien in den 1930er-Jahren schrittweise von wissenschaftlich glaubwürdigeren, explizit antirassistisch eingestellten Reformeugenikern abgelöst worden. ${ }^{2}$ Eine Betrachtung der Geschichte der internationalen eugenischen Bewegung stellt eine solche implizite Gleichsetzung von Rassismus und Pseudowissenschaftlichkeit infrage. ${ }^{3}$

1 Dieser Artikel basiert auf meinem Buch: Stefan Kühl 2014: Die Internationale der Rassisten. Aufstieg und Niedergang der internationalen eugenischen Bewegung im 20. Jahrhundert. Frankfurt a.M., New York: Campus; englische Ausgabe: Stefan Kühl 2013: For the Betterment of the Race. The Rise and Fall of the International Movement for Eugenics and Racial Hygiene. New York: Palgrave Macmillan. Es handelt sich um eine überarbeitete Fassung meines Artikels von 2004: Rassenforschung im Rahmen der internationalen eugenischen Bewegung. In: Peter Martin und Christine Alonzo (Hg.): Zwischen Charleston und Stechschritt. Schwarze im Nationalsozialismus. Köln: Dölling und Galitz, 495-507.

2 Vgl. Kenneth M. Ludmerer 1972: Genetics and American Society. Baltimore, London: Johns Hopkins University Press; Daniel J. Kevles 1986: In the Name of Eugenics. Genetics and the Uses of Human Heredity. Berkeley, Los Angeles: University of California Press.

3 Eine ausfuihrliche Schilderung einzelner Aspekte und auch umfassende Quellen- und Literaturnachweise finden sich in: Kühl 2014. 


\section{Eugenik und die Verwissenschaftlichung des Rassenbegriffs}

$\mathrm{Zu}$ Beginn des 20. Jahrhunderts wurden Eugenik und Rassenhygiene (die Begriffe wurden anfangs weitgehend synonym verwandt) als eigenständige Wissenschaft präsentiert. So forderten führende Eugeniker wie der Begründer der deutschen Rassenhygiene, Alfred Ploetz, der langjährige Vorsitzende der britischen eugenischen Gesellschaft, Leonard Darwin, und der führende US-amerikanische Eugeniker, Charles B. Davenport, dass die Eugenik sich an streng wissenschaftlichen Kriterien orientieren müsse. Besonders in der Anfangsphase versuchten alle drei, „eugenische Laien“ aus einflussreichen Positionen in den verschiedenen nationalen eugenischen und rassenhygienischen Organisationen zu verdrängen.

Die verschiedenen Strategien zur Internationalisierung der Eugenik mit der Gründung unterschiedlicher internationaler eugenischer Gesellschaften dienten dem Zweck, der Eugenik beziehungsweise der Rassenhygiene eine wissenschaftliche Basis zu geben. Schon die Bildung der Internationalen Gesellschaft für Rassenhygiene auf Initiative von Alfred Ploetz im Jahr 1907, aber besonders die Gründung des Permanent International Eugenics Committee 1912 sollten der Verwissenschaftlichung von Eugenik und Rassenhygiene dienen. Es wurde gehofft, durch den internationalen Austausch von Informationen über menschliche Vererbung, Rassenforschungen und Bevölkerungsentwicklungen das wissenschaftliche Kriterium der Intersubjektivität, also der Gültigkeit unabhängig von persönlichen Vorlieben und Wahrnehmungsmustern, zu erfüllen.

In den 1920er- und 1930er-Jahren versuchten die Eugeniker und Rassenhygieniker in der internationalen Bewegung die noch junge und fragile Wissenschaft der Eugenik gerade dadurch zu stabilisieren, dass sie die Rassenforschung intensivierten und professionalisierten. Die auch zur damaligen Zeit zunehmend wissenschaftlich überholten isolierten, anthropologischen Vermessungen von Schädeln und anderen menschlichen Körperteilen wurden durch Intelligenztests und international vergleichende Studien ergänzt beziehungsweise gar ersetzt. Die Gründung verschiedener Komitees zu Themen wie Rassenpsychiatrie, Rassenmischung oder Intelligenzforschung im Rahmen der International Federation of Eugenic Organizations, der Nachfolgeorganisation des Permanent International Eugenics Committee, hatte das Ziel, Anschluss an aktuelle wissenschaftliche Untersuchungen zu halten.

\section{Eugenische und rassenhygienische Forschung im Nationalsozialismus}

Wenn man sich die internationale Reaktion unmittelbar nach 1945 anschaut, dann könnte man annehmen, dass eine Gruppe von korrupten deutschen Wissenschaftlern 
alle ihre wissenschaftlichen Prinzipien über Bord geworfen hatte, um die nationalsozialistische Politik der „Rassenaufartung“ unterstützen zu können. Angesichts der Eindrücke von den nationalsozialistischen Massenmorden an religiös, ethnisch oder sozial definierten Gruppen, die zum Teil deutsche Wissenschaftler legitimiert hatten, wurden nach 1945 gerade die anthropologischen, genetischen und bevölkerungswissenschaftlichen Forschungsrichtungen im Nationalsozialismus zu einem Synonym für Unwissenschaftlichkeit. Diese Ansicht ist in einem gewissen Maß berechtigt: Es gab in Deutschland auch schon nach damaligen Standards wissenschaftlich kaum zu legitimierende Forschungen im Bereich der Anthropologie, Genetik oder Bevölkerungswissenschaft. So entsprachen Forschungen über einen vermeintlichen Gegensatz der genetischen Struktur von jüdischen und nichtjüdischen Deutschen nicht dem internationalen Niveau der damaligen Zeit. Aber dies ist lediglich die eine Seite der Medaille.

Die andere Seite ist, dass im Bereich der Eugenik, Psychiatrie, der genetisch orientierten Anthropologie und der Rassenforschung deutsche Wissenschaftler wirkten, deren wissenschaftliches Renommee weitgehend außer Frage stand. Obwohl Eugeniker und Rassenforscher wie die Humangenetiker Fritz Lenz und Freiherr Otmar von Verschuer sowie der Anthropologe Egon von Eickstedt eng mit einflussreichen nationalsozialistischen Stellen kooperierten, darf dies nicht darüber hinwegtäuschen, dass ihre wissenschaftlichen Forschungen jedenfalls in den 1930er-Jahren noch an die internationale Diskussion anschlussfähig waren. Beiträge der deutschen Humangenetik, biologischen Anthropologie, Bevölkerungsforschung oder Psychiatrie wurden auch in den 1930erJahren international rezipiert.

Aus dieser Sicht ist es nicht mehr so überraschend, dass die nationalsozialistische Politik der „Rassenaufartung“ die Anerkennung von Wissenschaftlern anderer Länder fand. Sicherlich gab es bei den nichtdeutschen Unterstützern der Rassenpolitik der Nationalsozialisten Wissenschaftler, die auf einem absteigenden Ast waren. Harry L. Laughlin zum Beispiel, einer der dominierenden Eugeniker in den USA, und der Rassenhygieniker Alfred Mjöen aus Norwegen hatten unübersehbar Schwierigkeiten, den aktuellen wissenschaftlichen Entwicklungen zu folgen. Darüber hinaus gehörten aber auch führende, wissenschaftlich weitgehend unumstrittene Eugeniker und Rassenhygieniker zu den Unterstützern der Nationalsozialisten. Im biologisch-anthropologischen Paradigma verankerte Eugeniker wie die anerkannten schwedischen Humangenetiker Herman Lundborg, Herman Nilsson-Ehle oder Torsten Sjögren zeigten sich von der Politik der Nationalsozialisten beeindruckt und verteidigten diese als wissenschaftlich fundiert.

Immer wieder wurde von den internationalen Unterstützern der nationalsozialistischen Rassenpolitik darauf verwiesen, dass die rassenhygienische Gesetzgebung der Nationalsozialisten auf Erfahrungen mit ähnlichen Gesetzen vor allem in den USA aufbauen 
konnte. Besonders die Verabschiedung des nationalsozialistischen Sterilisationsgesetzes nur wenige Monate nach der „Machtergreifung“ der Nationalsozialisten sei, so die Unterstützer, nur möglich gewesen, weil auf die Expertise US-amerikanischer Eugeniker zurückgegriffen werden konnte und sich die Erfahrungen mit Sterilisationsgesetzen in verschiedenen US-Bundesstaaten einbeziehen ließen. ${ }^{4}$

Wie breit die internationale Unterstützung für die Nationalsozialisten im Bereich der Eugenik, Rassenforschung und biologischen Anthropologie war, zeigt die Tatsache, dass die International Federation of Eugenic Organizations, die in den 1930er-Jahren eugenische Forschungsgesellschaften in über dreißig Staaten repräsentierte, die Maßnahmen zur Massensterilisationen von Behinderten in Nazi-Deutschland begrüßte. Auf der Konferenz der International Federation of Eugenic Organizations 1934 in Zürich legten die Vertreter der verschiedenen nationalen eugenischen Gesellschaften und Forschungsinstitute den „Regierungen der Welt" nahe, in "gleicher sachlicher Weise, wie dies bereits in einigen Ländern von Europa und Amerika geschehen ist, die Probleme der Erbbiologie, Bevölkerungspolitik und Rassenhygiene zu studieren und deren Ergebnisse zum Wohle ihrer Völker anzuwenden“ “ 5 Torsten Sjögren, nach 1936 Vorsitzender der International Federation of Eugenic Organizations, beabsichtigte sogar, im Namen der internationalen eugenischen Dachorganisation die Schirmherrschaft für einen internationalen Kongress für Eugenik und Rassenhygiene zu übernehmen, den die Nationalsozialisten 1940 in Wien durchführen wollten. ${ }^{6}$

\section{Die Kritik an der rassistischen Eugenik nach 1945}

Nach 1945 gab es in der Wissenschaft allgemein eine breite Abkehr von der Rassenforschung. Der Einfluss der Gene auf die geistigen Leistungen von „Rassen“ wurde von den meisten Humangenetikern bestritten. Die Mischung zwischen verschiedenen ethnischen Gruppen bezeichnete die überwältigende Mehrzahl der Wissenschaftler als unproblematisch. Darüber hinaus stellten zunehmend Sozialwissenschaftler, aber auch einzelne Naturwissenschaftler infrage, dass Gruppen überhaupt in genetisch verschiedene Rassen unterteilt werden könnten. „Rasse“ wurde also in der wissenschaftlichen Betrach-

4 Vgl. Stefan Kühl 2002: The Nazi Connection. Eugenics, American Racism, and German National Socialism. New York, Oxford: Oxford University Press.

5 IFEO 1934: Bericht der 11. Versammlung der Internationalen Föderation eugenischer Organisationen. Konferenzsitzungen vom 18. bis 21. Juli 1934 im Waldhaus Dolden. Zürich: 78 f. (Separatabdruck aus: Archiv der Julius-Klaus-Stiftung für Vererbungsforschung, Sozialanthropologie und Rassenhygiene. Bd. 10, Heft 1, 1935).

6 Sjögren an den Reichsinnenminister, 9.1.1939 (Kopie im Archiv des Max-Planck-Instituts für Psychiatrie in München, GDA 33). 
tung immer mehr zu einem „sozialen Mythos“, der lediglich dazu diente, bestimmte Gruppen zu diskriminieren.

Was war für diese grundlegende Veränderung in der Haltung der Wissenschaftler gegenüber der Rassenforschung verantwortlich? Es gab in den 1930er- und 1940erJahren kaum neuere Forschungen, die die rassistischen Forschungsansätze in der Eugenik und Anthropologie infrage gestellt haben. ${ }^{7}$ Die Abkehr von rassistischen Postulaten und implizit oder explizit rassistischen Forschungsansätzen hatte überwiegend politische Gründe. Dies zeigt besonders eindrücklich die Diskussion über die Statements der UNESCO zur Rassenfrage, mit denen die 1945 gegründete Kultur- und Wissenschaftsorganisation der Vereinten Nationen Forschung mit rassistischen Implikationen diskreditieren wollte. Wenngleich in den ersten beiden offiziellen Stellungnahmen 1950 und 1951 Unterschiede zwischen den geistigen Leistungen verschiedener Rassen bestritten und die Mischung zwischen verschiedenen ethnischen Gruppen als aus wissenschaftlicher Sicht unproblematisch bezeichnet wurden, verdeutlichen die gut dokumentierten internen Diskussionen, dass gerade einige der an der Ausarbeitung der Stellungnahmen beteiligte Naturwissenschaftler sich in Bezug auf die wissenschaftliche Fundierung ihrer Annahmen gar nicht so sicher waren. ${ }^{8}$

Die politische Stimmung nach 1945 hatte auch massive Auswirkungen auf die internationale eugenische Bewegung. Eugenik und Rassenhygiene waren durch die Nationalsozialisten so stark diskreditiert, dass ein wichtiger Teil der Eugeniker die Hoffnung aufgab, Eugenik und Rassenhygiene als eigenständige Wissenschaft jemals vollständig zu etablieren. Die Eugeniker, die unter anderem aus diesem Grund eine „Entwissenschaftlichung" der Eugenik propagierten, gehörten überwiegend den eugenischen Gesellschaften an, die sich in Rassenfragen zurückhielten. Gerade die hierin moderaten Vertreter der American Eugenics Society und der britischen Eugenics Society gaben nach 1945 das Motto aus, eugenisch relevante Forschung nur noch in den sich neu etablierenden Wissenschaftszweigen Humangenetik und Bevölkerungswissenschaft zu betreiben. Es wurde anerkannt, dass die Eugenik von vielen jenseits der Wissenschaft liegenden normativen Vorstellungen ausgehe und deswegen als eigene Wissenschaftsdisziplin keine Berechtigung habe. Die eugenischen Gesellschaften sollten zu politischen LobbyOrganisationen für die sich neu entwickelnden, „eugenisch nützlichen“Wissenschaften umgebaut werden.

Diese Rücknahme des mit der Eugenik und Rassenhygiene verbundenen Anspruchs lief der Strategie der an Rassenforschung orientierten Eugenik entgegen. Die Mehrzahl der an Rassenfragen orientierten Eugeniker hegte die Hoffnung, die Rassenforschung

7 Vgl. William B. Provine 1986: Geneticists and Race. American Zoologist. Jg. 26, 857-887.

8 Vgl. die intensiven Briefwechsel im Archiv der UNESCO, Paris (323.12 A 102). 
weiterhin über eine eigenständige eugenische Wissenschaft propagieren zu können. In dem Modell dieser unmittelbar nach dem Zweiten Weltkrieg relativ unbedeutenden Gruppe von Eugenikern waren Wissenschaft, Rassenforschung und Eugenik eng miteinander verkoppelt.

Da Eugenik und Rassenhygiene als eigenständige Wissenschaftsrichtung nach 1945 keine Lobby mehr hatte und die neu entstehenden Wissenschaftsdisziplinen der Humangenetik und Bevölkerungswissenschaft überwiegend von in Rassenfragen zurückhaltenden Eugenikern dominiert wurden, blieben für die an Rassenforschung interessierten Wissenschaftler vorrangig nur zwei wissenschaftliche Rückzugsgebiete: die humanbiologisch orientierte Anthropologie und die statistisch arbeitende psychologische Intelligenzforschung.

\section{Die „Renaissance“ der Rassenforschung in den 1960er-Jahren}

In den 1960er-Jahren kam es im Rahmen eines neu erwachten Interesses an Rassenfragen zur Bildung von wissenschaftlichen Gesellschaften und Zeitschriften. Diese „Renaissance“ der an Rassenfragen interessierten Eugenik hatte ihre Wurzeln weniger in der Humangenetik und der Bevölkerungswissenschaft, sondern vielmehr in der humanbiologisch orientierten Anthropologie und später in der statistisch ausgerichteten Intelligenzforschung. Genauso wenig wie die antirassistische Bewegung in den Wissenschaften nach dem Zweiten Weltkrieg vorrangig auf neue wissenschaftliche Erkenntnisse in der Humangenetik, Anthropologie oder Bevölkerungswissenschaft zurückzuführen gewesen war, spielten nun, in den 1960er-Jahren, neue wissenschaftliche Erkenntnisse eine Rolle. Hintergrund dieser „Renaissance“ war vielmehr die Aufhebung der Rassentrennung in den USA. ${ }^{9}$

In der politischen Auseinandersetzung um die Aufhebung der Rassensegregation in den USA spielte die UNESCO-Erklärung zur Rassenfrage vom Juli 1950 eine wichtige Rolle. Die Beweislast für die verschiedenen politischen Kräfte war nach 1945 unter anderem durch die UNESCO-Erklärung förmlich umgedreht worden. Während in den 1930er-Jahren Gegner der Rassentrennung gegen die Mehrheitsmeinung der Wissenschaft argumentieren und sich dadurch überwiegend auf moralische Argumente stützen mussten, standen jetzt die verbliebenen Vertreter der Rassentrennung unter immensem Legitimationsdruck, weil die wissenschaftliche Mehrheitsmeinung gegen sie gerichtet schien.

9 Vgl. William H. Tucker 2002: The Funding of Scientific Racism. Wickliffe Draper and the Pioneer Fund. Urbana, Chicago: University of Illinois Press, 65-130. 
Der Aufbau eines internationalen Netzwerks von Rassenforschern in den späten 1950er-, frühen 1960er-Jahren, das sich anfangs um die 1959 gegründete International Association for the Advancement of Ethnology and Eugenics und die heute noch existierende Zeitschrift Mankind Quarterly gruppierte, hatte das Ziel, in diese politische Diskussion unter dem Label der Wissenschaft einzugreifen. Während sich die Gruppe der Rassenforscher nach außen auf eine wertneutrale, rein wissenschaftliche Motivation für die Gründung der Zeitschrift und der eugenischen Dachorganisation zurückzog, geben die internen Briefwechsel interessante Einblicke in die politische Motivationslage: Die internationale eugenische Organisation unterhielt enge Kontakte zum NeonaziSpektrum in Europa und den USA. Die Zeitschrift Mankind Quarterly wurde von Wickliffe P. Draper finanziert, dessen Stiftung Pioneer Fund in den 1930er-Jahren die Verteilung von Propagandamaterial des nationalsozialistischen Rassenamtes in den USA organisiert hatte. Draper, der in den 1950er- und 1960er-Jahren einen erheblichen Teil der Rassenforschung in Europa und den USA finanzierte, knüpfte die Finanzierung des Mankind Quarterly an die Auflage, dass dieser seine „objektive Einstellung zu Rassenproblemen" beibehielte. ${ }^{10}$

Spezifisch für die Entwicklung der Rassenforschung nach 1945 war eine Immunisierung gegen jede Form von Kritik. Die eigene Position wurde als streng wissenschaftlich präsentiert. Kritiker aus unterschiedlichen Wissenschaftszweigen wurden als marxistisch motiviert und wissenschaftlich wenig seriös abgetan.

\section{Schlussbemerkung}

In der Auseinandersetzung mit der Eugenik genauso wie mit der biologisch orientierten Anthropologie muss selbstverständlich die Frage, inwiefern bestimmte Postulate und Forschungsansätze dieser Denkrichtungen an aktuelle Erkenntnisse der Humangenetik anschlussfähig sind, eine wichtige Rolle spielen. Eine Kritik, die sich auf wissenschaftliche Erkenntnisse der Humangenetik bezieht, kann mit dazu beitragen, rassistische Ansätze in der Wissenschaft aufzuzeigen.

Die historische Wissenschaftsforschung wählt jedoch einen anderen Ansatz. Ihre Stärke ist es, sowohl für ganze Wissenschaftsdisziplinen als auch für einzelne Forschungsprojekte nachzuweisen, wie einzelne wissenschaftliche Annahmen sozial konstruiert werden. Denn Forschungen mit rassistischen Implikationen verlieren viel von ihrer Bedrohlichkeit, wenn deutlich wird, wie entschieden sie Ausdruck von zeitlich und kontextuell gebundenen Arbeitshypothesen sind.

10 Vgl. Kühl 2002: 3-11; Kühl 2014: 205-232; Tucker 2002: 131-196. 